\title{
Identification of Error-Capturing Scan Cells in Scan-BIST With Applications to System-on-Chip
}

\author{
Chunsheng Liu, Member, IEEE, and Krishnendu Chakrabarty, Senior Member, IEEE
}

\begin{abstract}
We present a new partition-based fault-diagnosis technique for identifying error-capturing scan cells in a scan-BIST environment. This approach relies on a two-step scan chain partitioning scheme. In the first step, an interval-based partitioning scheme is used to generate a small number of partitions, where each element of a partition consists of a set of consecutive scan cells. In the second step, additional partitions are created using a previously proposed random-selection partitioning method. Two-step partitioning provides higher diagnostic resolution than previous schemes that rely either on random-selection partitioning or deterministic partitioning. We show via experiments that the proposed method requires only a small amount of additional hardware. The proposed scheme is especially suitable for a system-on-chip (SOC) composed of multiple embedded cores, where test access is provided by means of a TestRail that is threaded through the internal scan chains of the embedded cores. We present analytical results to characterize two-step partitioning, and present experimental results for the six largest ISCAS'89 benchmark circuits and two SOCs crafted from some of the ISCAS'89 circuits.
\end{abstract}

Index Terms-Diagnostic resolution, fault diagnosis, scan chain partitioning, system-on-chip.

\section{INTRODUCTION}

A S FEATURE sizes shrink and designs become more complex, built-in self-test (BIST) and scan design are gaining acceptance as industry-wide test solutions. The combination of scan design and BIST, commonly referred to as scan-BIST, is now especially common [1]. A scan-BIST technique typically applies a large number of pseudorandom patterns to the circuit under test via internal scan chains. The responses are then captured by the scan chain and compacted to a short signature in a multiple-input shift register (MISR). However, a drawback of this approach is that the signature in the MISR does not provide enough information for fault diagnosis, either to determine failing test vectors or to identify error-capturing (failing) scan cells.

The identification of error-capturing (failing) scan cells is useful for failure analysis because most of today's high-performance designs have shallow combinational logic depth between successive sequential stages. Once the information about failing

Manuscript received June 25, 2003; revised November 23, 2003. This research was supported in part by the National Science Foundation under Grants CCR-9875324 and CCR-0204077. A preliminary version of this paper was published in Proceedings of the Design, Automation, and Test in Europe (DATE) Conference, pp. 230-235, 2003. This paper was recommended by Associate Editor N. K. Jha.

C. Liu is with the Department of Computer and Electronic Engineering, University of Nebraska-Lincoln, Omaha, NE 68182-0572 USA.

K. Chakrabarty is with the Department of Electrical and Computer Engineering, Duke University, Durham, NC 27708 USA (e-mail: krish@ee.duke.edu).

Digital Object Identifier 10.1109/TCAD.2004.833620 scan cells is obtained, conventional non-BIST diagnostic techniques can be applied to further narrow down the set of candidate defect sites. For example, a diagnosis technique widely used in industry concentrates on the logic cones feeding the failing scan cells [5], [6].

For a scan-BIST scheme involving millions of vectors and tens of thousands of scan cells, the diagnosis and failure analysis time can be extremely high [1]-[4], [9]. Due to the large numbers of scan cells, it is often difficult to determine a small set of failing scan cells that can be used for failure analysis. Hence, there is a need for scan-BIST schemes that offer high diagnostic accuracy, i.e., they provide a small set of failing scan cells, without requiring excessive test-application time, processing time, and additional on-chip hardware.

Early work on failing scan cell identification was based on scan chain partitioning and the use of multiple BIST sessions. For example, a random-selection partitioning scheme is proposed in [5]. The scan chain is partitioned into nonoverlapping subchains, and each group (subchain) within the partition consists of a set of randomly-selected scan cells. Test patterns are applied to the circuit under test in multiple BIST sessions. The number of sessions is equal to the number of groups in the partition. In each session, a BIST signature is generated only for the scan cells that belong to the group within the partition corresponding to that session.

A single partition is generally insufficient for obtaining a small set of candidate failing scan cells, hence, the BIST sessions must be repeated for additional partitions. These partitions are randomly generated in [5]. Subsequent efforts to increase diagnostic accuracy and reduce test time have relied on binary search on a large number of scan cells [6], the principle of superposition [7], and deterministic partitioning [8]. However, an improvement in accuracy is often accompanied with a corresponding increase in hardware overhead, storage requirements for additional signatures, scan chain redesign, or restrictions on the size and number of partitions [6], [7]. In addition, all these previous approaches have been evaluated using a small number of errors that are randomly injected into the scan chains, and not using actual fault injection in benchmark circuits. Our premise here is that faults in real circuits do not produce errors in scan cells in a random fashion, and failing scan cells typically tend to be clustered.

The clustering of failing scan cells has an even greater impact on diagnosis for system-on-a-chip (SOC) designs that contain multiple embedded cores. Test access in such SOCs is often provided by means of a TestRail that is threaded through the internal scan chains of the embedded cores [12]. A spot manufacturing defect in a core-based SOC is likely to affect only a 
small number of cores, which implies that the failing scan cells can be expected to be clustered in the scan chains of the faulty cores. Known space diagnosis approaches that rely on random partitioning are therefore unlikely to be effective for the identification of failing scan cells in core-based SOCs.

We present a new scan-BIST approach for determining failing scan cells for fault diagnosis. The proposed approach relies on a two-step scan chain partitioning scheme. In the first step, an interval-based partitioning scheme is used to generate a small number of partitions, where each group within a partition (referred to as an interval) consists of a set of scan cells that are consecutively ordered in the scan chain. In the second step, additional partitions are created using the random-selection partitioning method. Two-step partitioning leads to higher diagnostic resolution than a scheme that relies only on random-selection partitioning, with only a small amount of additional hardware. Moreover, this approach is especially suitable for core-based SOCs since it targets clusters of failing scan cells. We improve the hardware architecture in [5] by implementing this two-step method with only two additional registers. Compared to more recent approaches based on deterministic partitioning [8], [13], two-step partitioning not only provides a superior diagnostic resolution, but it also obviates the need for scan chain redesign, which is often impossible in practice. The number of intervals can be changed as needed to achieve improved diagnostic resolution without changing the hardware.

The organization of this paper is as follows. In Section II, we describe the hardware architecture of this approach and present the corresponding scan-BIST architecture. In Section III, we present the motivation for the two-step partitioning scheme. In Section IV, we present analytical results to characterize the proposed two-step partitioning scheme. In Section V, we present experimental results for the single ISCAS' 89 benchmark circuits with a single scan chain each. In Section VI, we extend the method to multiple scan chains as well as to failing scan cell identification in core-based SOCs. We then present experimental results for two SOCs crafted from the ISCAS' 89 circuits, considering the scenarios of a single scan chain and multiple scan chains. We also compare the results with previous work based on random-selection partitioning [5], and more recent work based on deterministic partitioning [8], [13]. Finally, we implement the additional hardware for the different schemes using Synopsis tools and compare the corresponding area overheads.

\section{SCAN-Chain PARTITIONING}

In this section, we first review random-selection partitioning and present the scan-BIST architecture that is necessary for its implementation. We next review recent work on deterministic partitioning and discuss the advantages and drawbacks of this method. We then describe the proposed interval-based partitioning scheme and present the scan-BIST architecture needed to implement the proposed two-step scheme.

\section{A. Random-Selection Partitioning}

The identification of failing scan cells using random-selection partitioning proceeds as follows. First, the scan cells under diag-

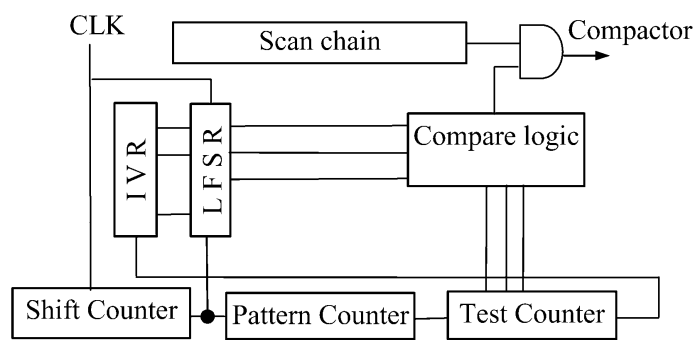

Fig. 1. Scan cell selection hardware used in random-selection partitioning scheme.

nosis are randomly selected and placed in a number of nonoverlapping groups [5]. This corresponds to the first partition. Multiple BIST sessions are then used for identifying failing scan cells, where each session corresponds to a group of scan cells within the partition. In the $i$ th BIST session, the MISR generates a signature only for the test responses captured by the scan cells in the $i$ th group of the partition. (The test responses for the other scan cells are masked.) In order to improve the diagnostic accuracy, a different partition is generated next, and the BIST sessions are repeated for this partition. This process is continued until a predefined number of partitions have been used, and signatures generated for the groups of scan cells within these partitions. Since there is overlap between the groups of scan cells in different partitions, the principle of inclusion and exclusion can be used to prune nonfailing scan cells. Additional pruning techniques can further refine the set of failing candidate scan cells [7].

Fig. 1 illustrates a scan cell selection hardware architecture implemented using an LFSR and initial value register (IVR) [5]. At the beginning of test application for each group within a partition, the LFSR is loaded from the IVR. The output of any $r$ stages of the LFSR can be regarded as a $r$-bit binary label associated with a specific scan cell. Clearly, if the number of groups in a partition is $b$, the length of test counter is $r=\left\lceil\log _{2} b\right\rceil$. On every shift, the label is compared with the current group number, i.e., the content of test counter. If a match occurs, the corresponding scan cell content is allowed to enter the compactor for signature analysis, otherwise it is masked. For the next group in the partition, i.e., test session, the LFSR is reloaded with the content of the IVR and a new group within the partition is set to selectively mask scan cells. Meanwhile, the test counter is incremented such that a different set of scan cells is compared for this group. At the end of each partition, the IVR is updated with the current value of the LFSR to create a different partition.

In the above random-selection scheme, scan cells in a group within a partition are selected randomly, hence, the elements of a group are likely to be scattered over the length of the scan chain. An alternative is to place in the same group a set of scan cells that are consecutively ordered in the scan chain, i.e., each group within a partition is an interval of scan cells and the partition consists of nonoverlapping intervals. One possible interval-based approach is to make all groups within a partition include the same number of scan cells, except for the boundary cases of the first or the last groups. However, this deterministic partitioning approach with fixed interval length can lead to significant routing overhead [8], [13]. 


\section{B. Deterministic Partitioning}

More recent work on partition-based diagnosis is based on the use of deterministic partitioning [8], [13]. In this approach, the scan cell groups within a partition are generated using modulo arithmetic such that the groups among the different partitions have minimum overlap. This can potentially lead to higher resolution when the overlapping partitions are fault-free. The hardware overhead required to generate deterministic partitions is comparable or even less than that for the random-selection scheme. It is shown in [8], [13] that compared to random-selection, deterministic partitioning reduces test application time by up to $25 \%$ for a given diagnostic resolution.

There are two possible hardware implementations of the deterministic partitioning scheme, referred to as monotonic and nonmonotonic in [8] and [13]. The former requires the positions of the scan cells in a group to increase monotonically. The latter implementation removes this restriction by dividing the scan chain into multiple smaller scan chains (tapped partitioning), where the size of the smaller scan chains is equivalent to the size of the scan cell group. However, it suffers from the need for circuit redesign and high routing overhead, especially when the number of groups is large. In many applications, e.g., when the circuit is an IP core, the scan chains are predesigned and cannot be changed, which renders the nonmonotonic approach infeasible. In addition, it is inflexible because once the size of the scan cell groups is determined, it cannot be subsequently changed, which does not allow refinement of the diagnostic resolution by varying the sizes of the scan cell groups.

The above deterministic partitioning scheme suffers from some additional limitations. For example, the size of the scan cell groups within a partition should be either a prime number or a power of two, and the size of the scan cell groups should be equal to the number of groups in a partition. The performance of the scheme is adversely affected if these conditions are not satisfied. For the experiments reported in [8], and [13], these restrictions were easily enforced since the authors only considered an ideal case, in which the length of the scan chain can be chosen arbitrarily and errors are randomly injected in the scan chain. However, in real circuits, it is very unlikely that these conditions can be satisfied; as a result, the performance of deterministic partitioning is expected to be worse than the ideal case. In addition, the proposed hardware is only suitable for the ideal case. If these conditions can not be satisfied, the hardware must be modified to correctly perform modulo arithmetic, which leads to additional overhead. We substantiate these arguments by means of experimental results later in this paper.

\section{Two-Step Partitioning}

We next propose a scan cell selection scheme that utilizes both random-selection and interval-based partitioning. The interval length, i.e., the number of scan cells in a group within a partition, is randomly generated for interval-based partitioning, and this leads to a simpler hardware implementation.

The selection hardware is sketched in Fig. 2. Compared to Fig. 1, this logic includes two additional registers, shift counter 2 and test counter 2 . The interval-based partitioning proceeds as

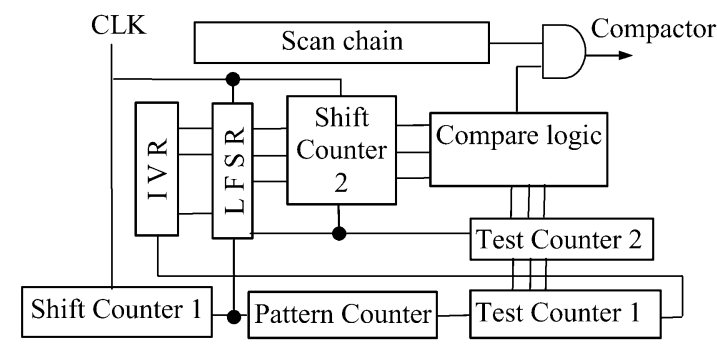

Fig. 2. Scan cell selection hardware used in two-step partitioning scheme.

follows. Initially, the LFSR is loaded with a precomputed seed in the IVR. The seed is associated with a number of bits from the LFSR and these bits can be viewed as a random number representing the length of the current interval. The seed is selected to ensure that a predefined number of groups generated by these bits can cover the entire scan chain. Usually, there exist a number of such seeds for a given circuit. In the beginning of each group, shift counter 2 is loaded with the current interval length from the LFSR. Simultaneously, test counter 1 is incremented and its content is transferred to test counter 2. At the end of each interval, test counter 2 is decremented. When test counter 2 reaches 0 , the compared logic outputs 1 . Therefore, the contents of the scan cells are selected and shifted into the compactor; this continues until shift counter 2 also reaches 0 . The procedure continues for all the patterns in the test and the signature collected in the compactor is the response for the current interval in the partition. This process then iterates until all the intervals in the partition are handled. To use more than one interval-based partition, the LVR is initialized with a different seed and the above procedure is repeated. If the size of the largest scan cell group is $N_{p}$, which is usually much smaller than the length of scan chain, and the number of scan cell groups within a partition is $P$, then the number of flip-flops needed by the two additional registers is $\left\lceil\log _{2} N_{p}\right\rceil+\left\lceil\log _{2} P\right\rceil$.

We next present an example to illustrate interval-based partitioning. Suppose we have a scan chain with 16 scan cells, and the first partition includes four groups of scan cells. We also assume that the three selected bits of the LFSR, which is seeded by the IVR, generate four pseudorandom patterns 101, 110, 011, and 010. These correspond to intervals of length $5,6,3$, and 2 , respectively. The content of test counters 1 and 2 are initially both 0 . Therefore, the compare logic enables the AND gate to transfer the content of the scan cells to the compactor. Since shift counter 2 is next set to 5 and decremented thereafter, the scan cells 1-5 are scanned out to the compactor. After all the BIST patterns are applied, test counter 1 is incremented, hence, test counter 2 is now initialized with the value 1 . The LFSR is reloaded with the same seed from the IVR and once again shift counter 2 is initialized with the value 5 . When shift counter 2 is decremented to 0 by the shift clock, a carry signal drives the LFSR to shift once and the next random output 6 is loaded into shift counter 2 . Meanwhile, the content of test counter 2 is decremented to 0 . As a result, the compared logic outputs a 1 and the scan cells from positions 6 to 11 transfer their contents to the into compactor. The remaining two intervals are handled in a similar fashion.

After test application using a small number of interval-based partitions, the random-selection scheme is used to create the re- 
maining partitions. This can be done by simply disabling shift counter 2 and test counter 2 or bypassing them. An important advantage of this scan-BIST architecture is that it can readily incorporate various other partitioning schemes without hardware modifications. Moreover, the entire diagnosis process can be carried out without interruptions or manual intervention. This is in contrast to the adaptive scheme in [6], where test application must be frequently interrupted to execute a binary search procedure.

\section{Motivation FOR Two-STEP PARTITIONING}

Prior work on the identification of failing scan cells is based on random-selection partitioning, which is especially effective if the failing scan cells are uniformly distributed across a scan chain. However, in practice, faults in a circuit are likely to lead to clustered failing scan cells, thereby invalidating the uniform distribution assumption implicit in random-selection partitioning. An error due to a fault can only be captured by a set of scan cells that lie within the fault cone, i.e., the scan cells that can be reached by a sensitized path from the fault site. The locations of these error-capturing scan cells in the scan chain depend on the scan chain ordering, but there is nevertheless a clear dependence between the circuit structure and the distribution of failing scan cells. Therefore, errors caused by a fault are restricted to a small segment of the scan chain. If multiple faults exist in the circuit, the fault cones may either be nonoverlapping, leading to nonoverlapped segments of failing scan cells, or the fault cones may lead to overlapped segments of failing scan cells, which can be viewed as one expanded segment.

An obvious drawback of random selection is that since scan cells in any group of a partition are selected randomly, a segment associated with a fault cone is often fragmented, and error-capturing scan cells in a segment are placed in different groups of a partition. Since a single failing scan cell in a group renders all the scan cells in that group to be candidate fails, random selection decreases the likelihood that a group of nonfailing scan cells will be pruned from the candidate set. Note however that this problem is severe only for the first few partitions. Once the candidate set is reduced through pruning, random selection is extremely effective in reducing the candidate set further.

In contrast to random-selection partitioning, interval-based partitioning leads to significant pruning for the first few partitions. Since scan cells are grouped into nonoverlapping intervals, a segment associated with a fault cone can be covered by a small number of consecutive intervals. This can be done by carefully selecting the number of groups within a partition and the seed used to generate interval length. As a result, candidate failing scan cells are restricted to these intervals and this can lead to a significantly smaller set of failing candidates. We have seen in our experiments that one or two interval-based partitions are usually adequate to reduce the candidate size considerably, following which random selection can be employed for further pruning.

We next highlight the effectiveness of interval-based partitioning through the example in Fig. 3. For the sake of simplicity, we use one of the smaller ISCAS' 89 benchmark circuits, namely s953. Suppose it is implemented as a full-scan circuit
True failing scan cells: 2

0000110000000000000000000000000000000000000000000000

Interval-based partitioning:

Group 1: 1-4

Group 2: 5-12

Group 3: 13-28

Group 4: 29-52

No. of suspect failing scan cells: 8

0000111111110000000000000000000000000000000000000000

Random-selection partitioning:

Group 1: $14,15,18,26,35,36,37,47,52$

Group 2: 4,7,9,12,16,19,24,27,31,38,42,48,50

Group 3: 3,6,8,11,13,17,23,25,30,34,41,46,49,51

Group 4: 1,2,5,10,20,21,22,28,29,32,33,39,40,43,44,45

No. of suspect failing scan cells: 30

1110110101101000100111101001110111000011101111001010

Fig. 3. Candidate failing scan cells determined using one partition based on interval-based and random-selection partitioning, respectively.

with a single scan chain. We inject a single stuck-at fault into this circuit and observe the number of failing scan cells for a randomly-chosen input pattern that detects this fault. For this example, there are two failing scan cells, denoted by ' 1 ' in the figure. (The nonfailing scan cells are denoted by ' 0 '.)

We then generate one partition each using the interval-based method and the random-selection method, respectively. The partition in each case consists of 4 groups. The figure shows the corresponding partition contents and the candidate failing scan cells determined from pass/fail of each group within the partition. Interval-based partitioning yields much better resolution than random-selection partitioning. This is because the intervalbased method places the two failing scan cells in the same group, while the random-selection method disperses them to two different groups.

Despite the obvious advantage of interval-based partitioning highlighted by Fig. 3, random selection is superior in one important aspect. By providing greater randomness, it outperforms interval-based partitioning when a large number of partitions are employed. For example, if the first and the last scan cell in the (ordered) scan chain are failing, then they always belong to two different intervals, irrespective of the number of partitions generated. However, in a random-selection partition, they may be included in the same group. Therefore, we combine the benefits offered by the two approaches by adopting a two-step procedure. We first create a small number of partitions using interval-based method. This allows us to achieve rapid coarse-grained resolution. Next, the random-selection method is used to generate other partitions, which allows us to achieve fine-grain resolution. For the sake of simplicity, we use only one interval-based partition for the two-step method in our simulations, even though we have observed that in some cases, the use of more interval-based partitions leads to higher diagnostic resolution.

\section{ANALyTiCAl Results}

In this section, we analyze the two-step partitioning scheme using a probabilistic method. We first review the technique used to analyze the random-selection scheme in [5], and then extend the analysis to make it applicable to the two-step scheme. 
The performance of a partitioning scheme $S$ can be measured by the average number $F_{S}$ of fault-free scan cells that declared as suspect failing scan cells after diagnosis. A smaller value of $F_{S}$ indicates a higher diagnostic resolution. The ideal value of $F_{S}$ is 0 , meaning that only the true failing scan cells are included in the candidate list [5].

For the random-selection scheme, $F_{r}$ can be estimated by the equation $F_{r}=(n-k)\left(1-(1-1 / b)^{k}\right)^{c}$, where $n$ is the length of scan chain, $k$ is the number of true failing scan cells, $b$ is the number scan cell groups in a partition and $c$ is the number of partitions. The equation can be interpreted approximately as follows. For any fault-free scan cell, $1 / b$ can be viewed as the probability that it belongs to the same scan cell group with one of the $k$ faulty scan cells, and $1-1 / b$ is the probability that it is not in the same scan cell group with that faulty scan cell. Then, $(1-1 / b)^{k}$ is the probability that this scan cell does not belong to the same group with any faulty scan cell, meaning that it can be declared to be faulty-free. Consequently, $1-(1-$ $1 / b)^{k}$ is the probability that a given fault-free scan cell cannot be identified as fault-free at the end of a partition, therefore, it has to be included in the candidate list. Finally, the probability that a given fault-free scan cell cannot be declared as fault-free at the end of $c$ partitions is $\left(1-(1-1 / b)^{k}\right)^{c}$. Since all the scan cells are randomly chosen to form the groups, they can be viewed as identical with respect to the probability of being included in the candidate list. As a result, the average number $F_{r}$ of fault-free scan cells that cannot be declared to be fault-free is

$$
F_{r}=(n-k)\left(1-\left(1-\frac{1}{b}\right)^{k}\right)^{c}
$$

A similar analysis can be applied to the interval-based partitioning scheme. However, a major difference here is that the fault-free scan cells cannot be viewed as identical with respect to the probability of being included in the suspect candidate list. Clearly, the fault-free scan cells that are closer in position to the failing scan cells are more likely to be included in the same group with the failing scan cells, hence they are more likely to be included in the suspect candidate list than the fault-free scan cells that are relatively far in position to the failing scan cells. For example, if a fault-free scan cell is adjacent to a failing scan cell, then it is very likely that the two scan cells will always be covered by the same group. As a result, this fault-free scan cell is very difficult to be identified as fault-free, and it will most likely be included in the candidate list.

Since the fault-free scan cells cannot be regarded as identical and the position and length of the intervals are randomly generated, it is difficult to estimate the average number $F_{i}$ of fault-free scan cells in the candidate list. In order to simplify the analysis, we partition the entire scan chain (with $n$ scan cells) into $b$ subsections with a uniform length $n / b$. All the $k$ failing scan cells fall into $s$ subsections $(s \leq b$ and $s \leq k)$ and a smaller value of $s$ indicates that the failing scan cells are more clustered. We can classify all the $n-k$ fault-free scan cells into two categories. If a fault-free scan cell is in the $s$ subsections containing failing scan cells, the probability that it belongs to the same group with a failing scan cell is very likely to be higher than $1 / b$, which is obtained in the random case. We incorporate a weighting factor
$P_{h}\left(P_{h}>1\right)$ and adjust this probability to be $P_{h} / b$. Similarly, if a fault-free scan cell is not in the $s$ subsections, the probability that it belongs to the same group with a failing scan cell is very likely to be lower than $1 / b$, which can be estimated as $P_{l} / b\left(P_{l}<1\right)$. The probability that a given fault-free scan cell cannot be declared as fault-free at the end of $c$ partitions is therefore $\left(1-\left(1-P_{h} / b\right)^{k}\right)^{c}$ and $\left(1-\left(1-P_{l} / b\right)^{k}\right)^{c}$, respectively, for the two categories. Since the number of scan cells in the two categories are $((n-k) s / b)$ and $((n-k)(b-s) / b)$, respectively, the average number $F_{i}$ of fault-free scan cells that cannot be declared as fault-free at the end of $c$ partitions in the interval-based partitioning is

$$
\begin{aligned}
F_{i}=\frac{(n-k) s}{b}( & \left.1-\left(1-\frac{P_{h}}{b}\right)^{k}\right)^{c} \\
& +\frac{(n-k)(b-s)}{b}\left(1-\left(1-\frac{P_{l}}{b}\right)^{k}\right)^{c}
\end{aligned}
$$

Although this is admittedly a coarse-grained estimation, the underlying approximation appears to be reasonable, and the analytical results match well with the experimental results.

From the above analysis, we can easily determine the average number $F_{t}$ of fault-free scan cells included in the candidate set for the two-step scheme. Since partitions are mutually independent, the results obtained from the interval-based partition can be directly applied to the subsequent random-selection partitions. If we use interval-based scheme for the first $i$ partitions and random-selection scheme for the remaining $c-i$ partitions, $F_{t}$ can be simply obtained by combining (1) and (2)

$$
\begin{aligned}
F_{t}= & \left(\frac{(n-k) s}{b}\left(1-\left(1-\frac{P_{h}}{b}\right)^{k}\right)^{i}\right. \\
& \left.+\frac{(n-k)(b-s)}{b}\left(1-\left(1-\frac{P_{l}}{b}\right)^{k}\right)^{i}\right) \\
& \times\left(1-\left(1-\frac{1}{b}\right)^{k}\right)^{c-i} .
\end{aligned}
$$

In Fig. 4(a) and (b), we illustrate the analytical results. We choose some typical values as the parameters in the calculation as shown in the figure. We select the length of scan chain $n=1000$ and 5000 in (a) and (b), respectively. The number of scan cell groups $b=16$, the number of failing scan cells $k=20$, number of partitions $c=8$. We also assume that the failing scan cells are clustered in $s$ subsections and the factors $P_{h}=2.5$ and $P_{l}=0.4$, respectively. The results in Fig. 4 clearly show that initially, the interval-based partitioning yields a smaller $F_{I}$ (compared to $F_{R}$ ), hence it provides better resolution. As the number of partitions increases, the random-selection scheme outperforms the interval-based scheme. This supports our claim that the interval-based partitioning is more suitable for coarse-grained diagnosis and the random-selection is suitable for fine-grained diagnosis. It is also consistant with our experimental results on ISCAS benchmarks shown in the next section. However, we observe that in all cases, the two-step partitioning yields the best resolution. Calculations using a number 


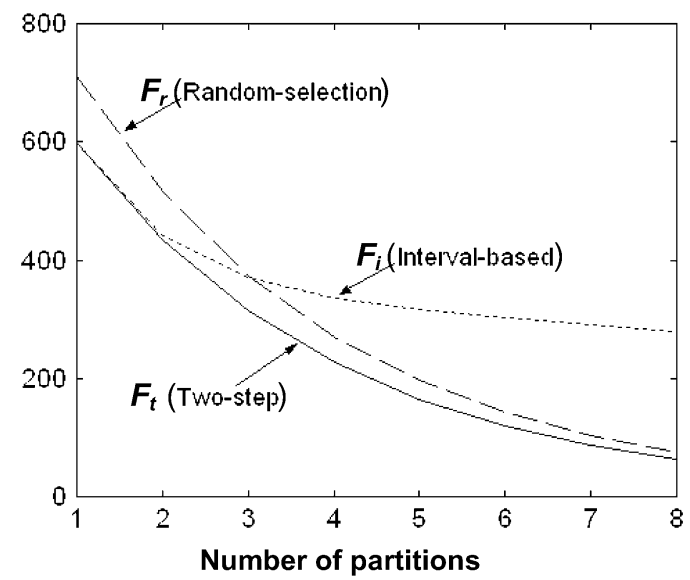

(a)

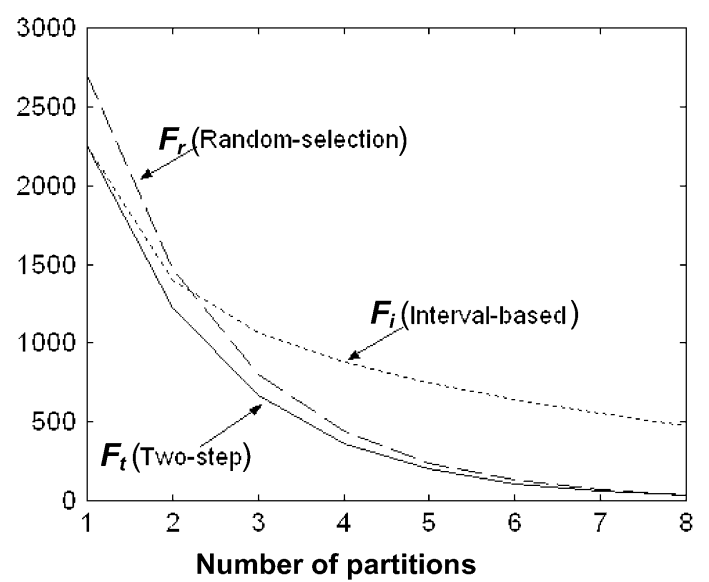

(b)

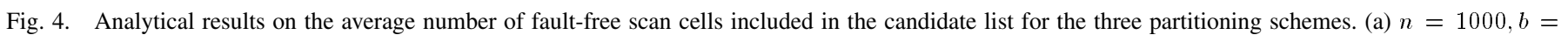
$16, k=20, c=8, s=6, P_{h}=2.5, P_{l}=0.4$. (b) $n=5000, b=64, k=50, c=8, s=20, P_{h}=2.5, P_{l}=0.4$.

of other values of the parameters yield similar results; these are not presented here for reasons of conciseness.

The analytical results can provide a guideline for the set-up of partitioning hardware, i.e., to determine the values of $b, c$, etc., such that the resulting resolution $F_{t}$ is satisfactory and the test application time associated with $b$ and $c$ is reasonable. Unfortunately, almost all the values of variables in (3) are not known a priori and must be determined empirically for each circuit. A possible three-step procedure is outlined as follows. In the first step, we use (1) to estimate the values of $b$ and $c$. We select an acceptable value for $F_{r}$ and an arbitrarily-chosen value of $k$ ( $k$ is unknown before diagnosis). The value of either $b$ or $c$ can be obtained using (1) if the other is set. For example, if we set the value of $b$, then $c$ can be determined from the following:

$$
c=\frac{\left(\ln F_{r}-\ln (n-k)\right)}{\ln \left(1-\left(1-\frac{1}{b}\right)^{k}\right)}
$$

The parameter $b$ can be determined in a similar way if we set the value of $c$. In the second step, we use these values to set up the hardware and run several more diagnostic samples. This is a conservative setup and the objective resolution $F_{r}$ used here is an overestimate, because the two-step scheme can provide a much better resolution. Therefore, the resulting resolution $F_{t}$ can be expected to be more than the threshold of acceptable resolution. We then use these empirical results to obtain $F_{t}, k$ and $s$, e.g., by taking the average values over all the samples. We can also estimate the weighting factors $P_{h}$ and $P_{l}$ using the empirical results. In the last step, we put the values of all these variables back into (3) and recalculate either $b$ or $c$. The second and third steps can be repeated iteratively to further refine the values of $b$ and $c$ to guarantee a satisfactory diagnostic resolution without burdening the diagnosis procedure with excessive test application.

We note that the two-step scheme is particularly suitable for this procedure where the values of $b$ and $c$ need to be changed frequently. In both random-selection and two-step methods, $c$ can be changed easily by stopping the clock after a number of partitions. However, the two-step scheme can also change the number of scan cell groups $b$ easily by simply reseeding the
LFSR, which is not possible in random-selection. In the deterministic schemes of [7] and [8], the change in these values requires hardware changes; therefore, it is often not feasible. This is potentially a serious drawback since if the pre-estimated partition parameters $b$ and $c$ do not match the specific circuits well enough, the lack of flexibility may lead to low diagnostic resolution and excessive test-application time.

\section{EXPERIMENTAl Results ON Single-Benchmark CIRCUITS}

In this section, we present simulation results for the full-scan versions of the ISCAS' 89 benchmark circuits. We assume that each of these circuits contains a single scan chain. For each circuit, we inject 500 single stuck-at faults. In contrast to earlier work based on random error injection in scan cells, we inject faults into the circuits and examine their effect on the errors captured in scan cells. If we use $N_{C}$ and $N_{T}$ to represent the number of candidate failing scan cells and the number of true failing scan cells, respectively, then the diagnostic accurancy in terms of diagnostic resolution DR can be calculated as follows:

$$
\mathrm{DR}=\frac{\sum_{\forall \text { fault } f} N_{C}-\sum_{\forall \text { fault } f} N_{T}}{\sum_{\forall \text { fault } f} N_{T}} .
$$

The parameter DR is a measure of the average number of faultfree scan cells that are included in the candidate set. A low value of DR denotes high diagnostic accuracy. In the ideal case, DR has a value of zero, which means the candidate set includes all the actual failing scan cells and no fault-free scan cells.

First, in Fig. 5, we present DR results for a small circuit s953 when the number of partitions is varied from 1 to 8 . The DR values are indicated at the top of the bars. We consider a BIST test session consisting of 200 pseudorandom patterns. It can be seen that when the number of partitions is small, the intervalbased method provides better resolution than random-selection. However, for a larger number of partitions, the random-selection method yields higher resolution. As discussed in Section IV and shown in Fig. 4, this result is expected since random selection provides fine-grained resolution for a larger number of partitions. In all the cases, the two-step method shows the best 


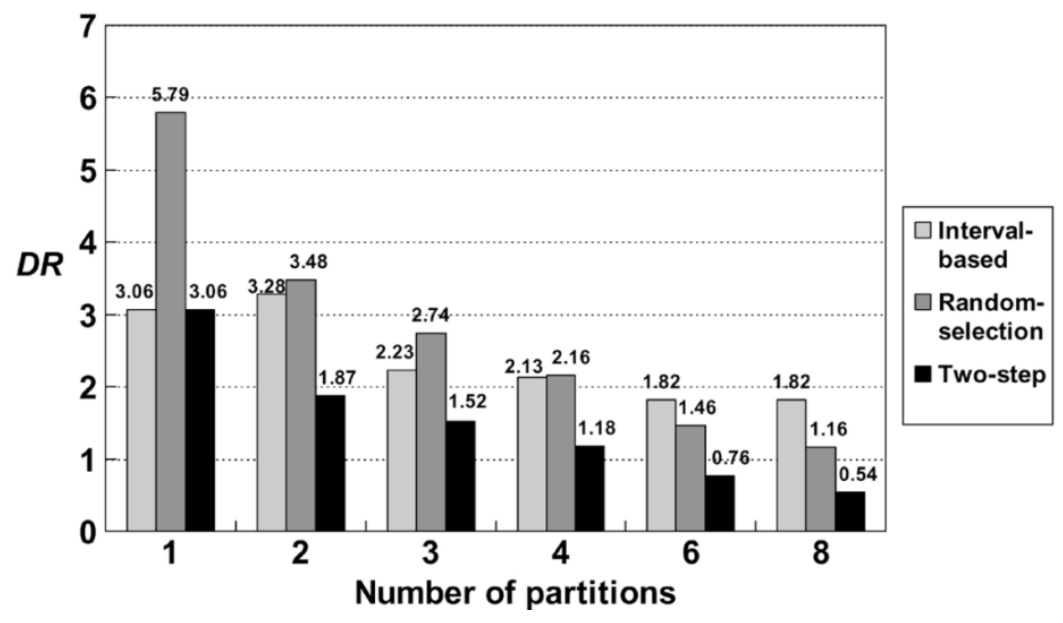

Fig. 5. Diagnostic resolution obtained for s953 with varying number of partitions and different partitioning schemes.

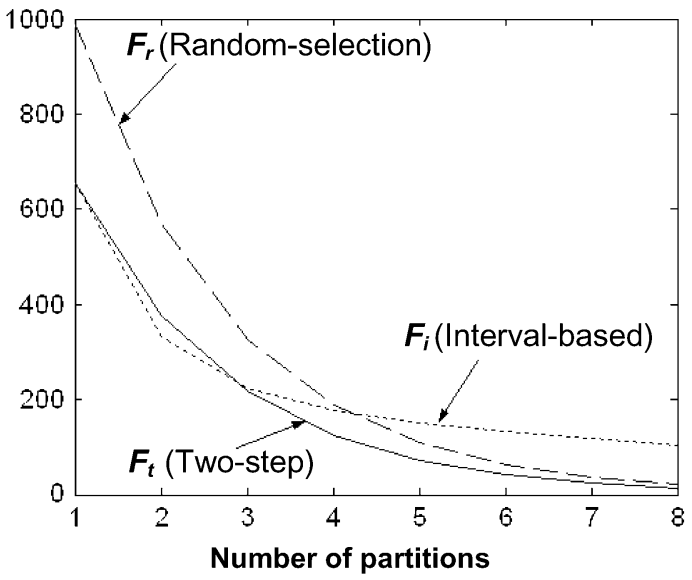

(a)

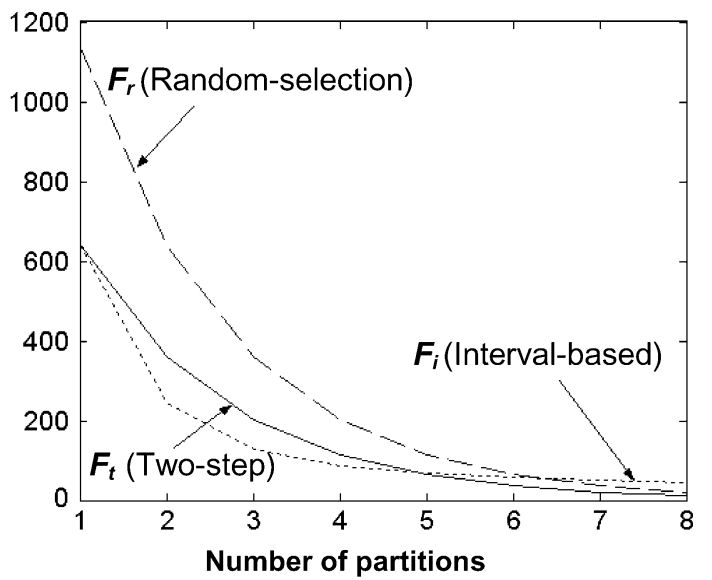

(b)

Fig. 6. Experimental results on the average number of fault-free scan cells included in the candidate list for the three partitioning schemes for (a) $\mathrm{s} 38417: n=$ $1742, b=32, k=27, c=8, s=5, P_{h}=2.5, P_{l}=0.4$. (b) s35932: $n=2048, b=32, k=27, c=8, s=2, P_{h}=2.5, P_{l}=0.4$.

resolution, and its DR value is almost $50 \%$ smaller than that for random selection.

Next, we apply the analysis method of Section IV to two of the larger benchmark circuits, namely s38417 and s35932. Following the procedure described in Section IV, we run the simulation over a number of $(>100)$ diagnostic runs with different faults injected each time and with the refinement of the partitioning parameters. In Fig. 6, we report the resulting values of the relevant variables and the average number of fault-free scan cells included in the failing scan cell list by the three different partitioning schemes shown in Fig. 4. It can be seen that these experimental results nicely match the analytical results of Fig. 4. (There is a slight offset in the results due to the difference in the parameter values.) Although the interval-based scheme outperforms the two-step scheme at some points when the number of partitions is small, i.e., low diagnostic resolution is acceptable, the two-step scheme emerges as the best approach when all partitions are eventually applied. These results demonstrate that the analysis in Section IV can serve as a valuable guideline for estimating the performance and the hardware configuration of the partitioning schemes.

In Fig. 7, we present simulation results for the six largest ISCAS' 89 benchmarks using the deterministic test patterns from Mintest [14] and pseudorandom test patterns, respectively. Since fault simulation for a large number of test patterns is extremely time-consuming, here we apply only 10000 pseudorandom patterns. The fault efficiencies (i.e., the percentage of detectable faults that are detected) for different benchmarks are $100 \%$ for Mintest ATPG test patterns and vary from $90 \%$ to $99 \%$ for pseudorandom test patters. We use a primitive-polynomial LFSR of degree 16 to create partitions. We perform simulation for the three schemes: random-selection, deterministic and two-step. We list results for both monotonic and nonmonotonic implementations, even though the latter leads to high routing overhead. In order to make an objective comparison, we use the same number of partitions ( 8 for s9234 and 16 for the others) and the same number of scan cell groups ( 8 for s9234, s15850 and s13207, and 16 for the others) within each partition for all methods, hence, these methods require the same test application time.

Two-step partitioning provides greater diagnostic accuracy than random selection for every circuit. It also outperforms deterministic partitioning in most cases. For the larger circuits, the DR values are reduced by as much as $80 \%$ compared to random selection and even more compared to deterministic partitioning. The nonmonotonic scheme provides slightly better results than 


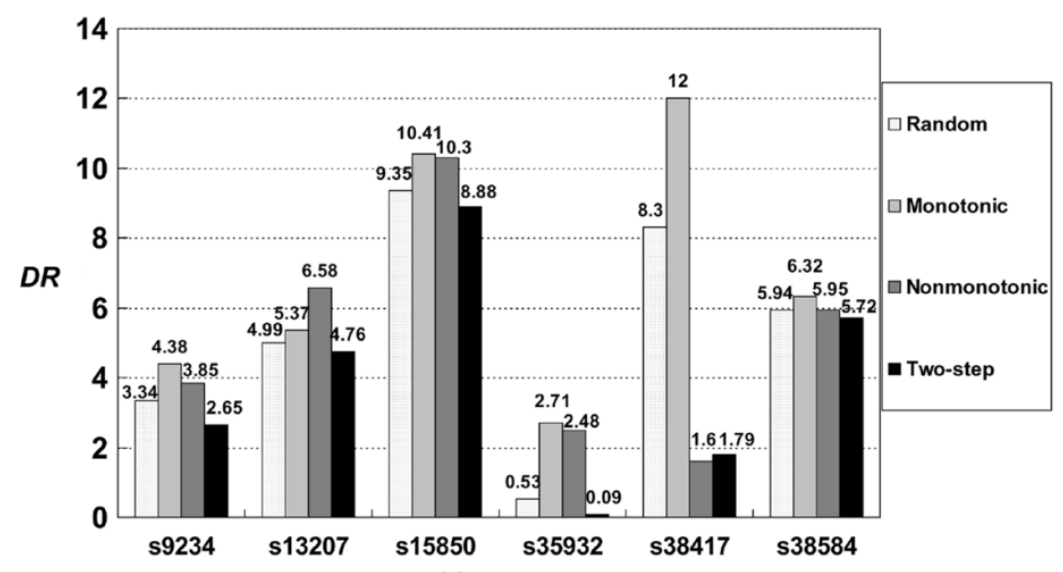

(a)

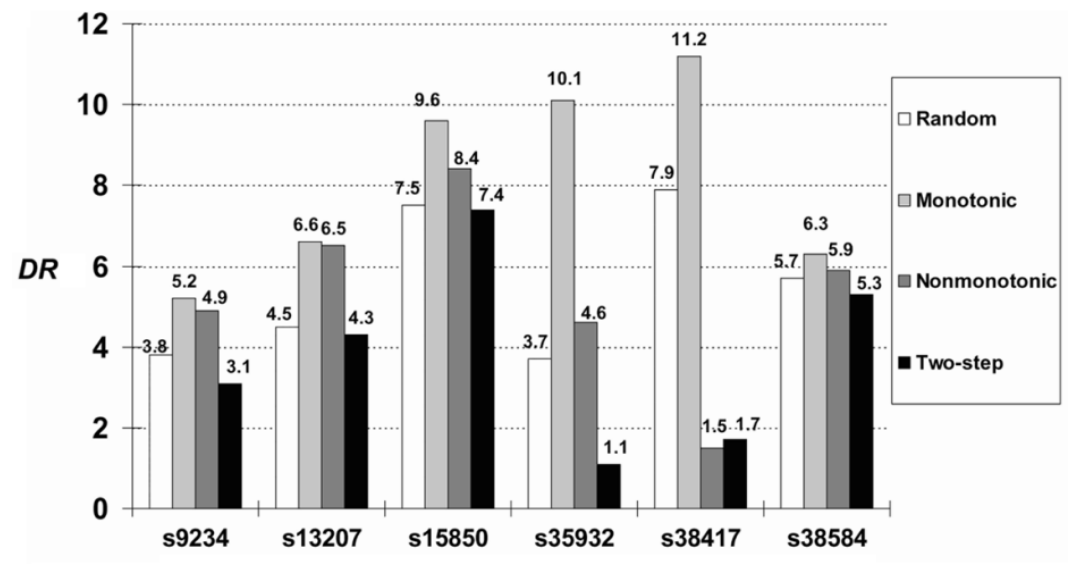

(b)

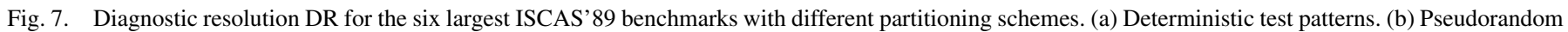
test patterns.

the two-step scheme in only one case because of the irregular interval lengths generated in the latter. The results for the proposed method for this case can be improved by searching for more effective seeds for the LFSR. However, as we will show in Fig. 14, the two-step scheme can reduce the test application time by $50 \%$ over the nonmonotonic scheme when the desired diagnostic resolution is higher. The deterministic schemes yield worse results than random selection in many cases because in these benchmark circuits it is not possible to satisfy all the conditions with respect to the group size and the number of groups. We also note that the DR values here are larger than those obtained by random error injection using a small number of errors reported in [5]. This is because in a real circuit, some faults may cause a large number of failing scan cells that make partitioning and pruning less effective.

Once fault simulation is completed, the simulation of the twostep scheme consumes little CPU time. On a Sun Blade 1000 workstation with a $750 \mathrm{Mhz}$ processor and $1 \mathrm{~GB}$ memory, the simulation for each circuit can be finished within a few seconds of CPU time.

The pruning technique of [7] can be combined with two-step partitioning to obtain even higher diagnostic accuracy. In Fig. 8, we list the diagnostic resolution obtained by applying the postprocessing pruning technique proposed in [7] on top of the results shown in Fig. 7(a). It can be seen that compared to Fig. 7(a), the resolution is significantly improved for all the methods, but the two-step scheme remains to be the one with best performance in most cases. For the sake of conciseness, in the subsequent experimental results, we assume that no pruning is applied.

The advantages of the two-step partitioning scheme are not limitted to single stuck-at faults. In Fig. 9, we show simulation results for some benchmarks using various other types of faults. In Fig. 9(a), we show the results averaged over 100 gate replacement design errors, including the errors of NAND $\rightarrow$ NOR replacement, NOR $\rightarrow$ NAND replacement and gate polarity change. In Fig. 9(b), we show the average results for 100 bridging faults, including AND-bridging, OR-bridging, and XNOR-bridging. It can be seen that the two-step scheme provides a significantly better resolution than any other scheme. In many cases, the DR value for the two-step scheme is zero, which indicates perfect resolution.

Once the failing scan cells are identified, various conventional diagnostic methods can be applied to locate the defect sites in the circuit, which is the eventual goal of diagnosis. A smaller number of failing candidate scan cells can facilitate this process and lead to a smaller number of candidate defect sites. In Table I, we show the simulation results for s953. We first inject two faults, a gate replacement fault and a bridging fault, in the circuit one at a time. We then use different partitioning schemes to identify a set of failing scan cells and list the results in Columns 2 to 5 of Table I. Finally, we apply a simple diagnostic method 


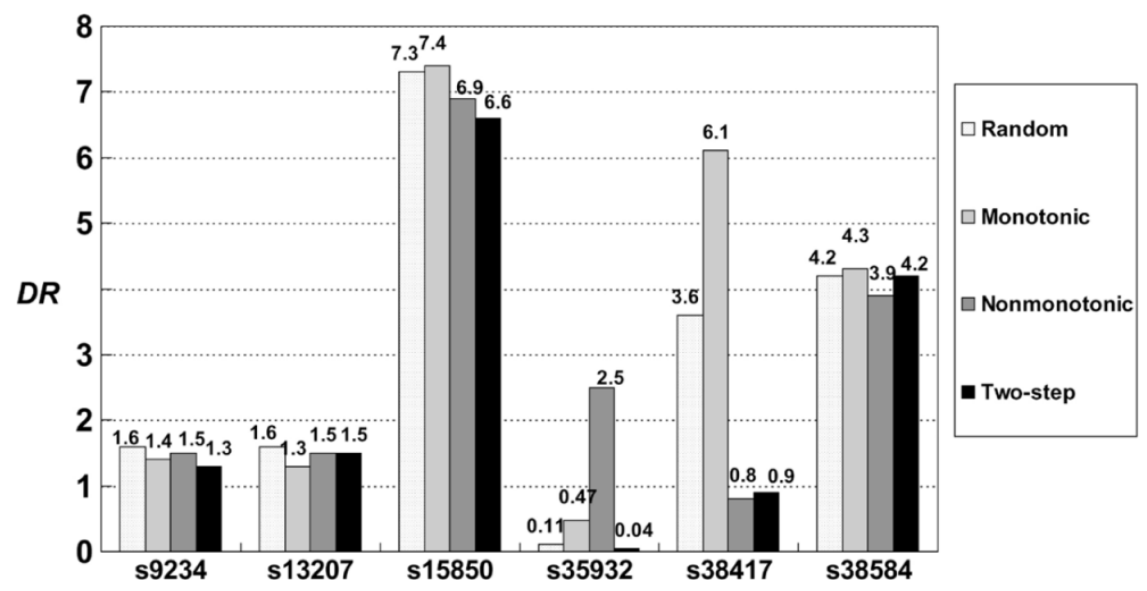

Fig. 8. Diagnostic resolution DR of the six largest ISCAS' 89 benchmarks with different partitioning schemes with pruning procedure in [7].

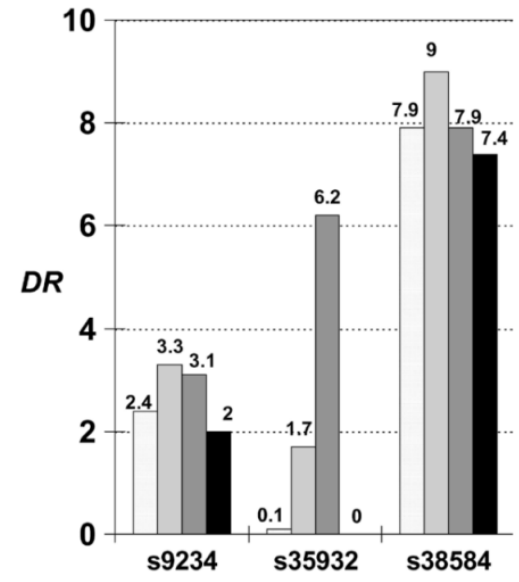

(a)

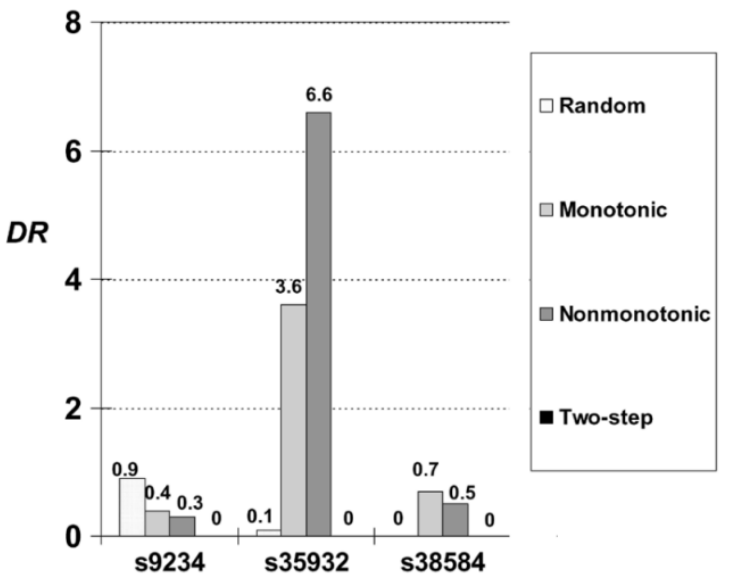

(b)

Fig. 9. Diagnostic resolution DR for the ISCAS'89 benchmarks with design errors (gate replacement) and bridging faults. (a) Gate replacement errors. (b) Bridging faults.

TABLE I

DiAgNosis RESUlTS FOR Two FAULTS FOR S953 USING LOGIC CONES

\begin{tabular}{c|c|c|c|c|c|c|c|c}
\hline \multirow{2}{*}{ Fault } & \multicolumn{2}{|c|}{ Number of candidate error-capturing scan cells } & \multicolumn{3}{c}{ Number of candidate defect sites } \\
\cline { 2 - 8 } & $\begin{array}{c}\text { Random } \\
\text { selection }\end{array}$ & $\begin{array}{c}\text { Two- } \\
\text { step }\end{array}$ & Monotonic & $\begin{array}{c}\text { Non- } \\
\text { monotonic }\end{array}$ & $\begin{array}{c}\text { Random } \\
\text { selection }\end{array}$ & $\begin{array}{c}\text { Two- } \\
\text { step }\end{array}$ & Monotonic & $\begin{array}{c}\text { Non- } \\
\text { monotonic }\end{array}$ \\
\hline 1 & 15 & $4^{*}$ & 12 & 5 & 287 & 187 & 229 & 215 \\
\hline 2 & 8 & $2 *$ & 5 & 3 & 209 & 141 & 160 & 182 \\
\hline
\end{tabular}

*All the candidates are true failing scan cells.

using logic cones to determine a set of candidate defect sites. Each failing scan cell is associated with a logic cone, which consists of all the nodes that can possibly feed this scan cell. This simple method takes the union of all logic cones and reports the number of nodes in the union, i.e., the size of candidate set of defect site, in the last four columns of Table I. It can be seen that the two-step scheme provides the smallest set of candidate sites. Since this is a straightforward defect-location method that does not use additional information such as failing test vectors, the numbers in Table I are quite large for all the methods. In practice, the set of defect sites can be reduced using additional information. Here we only present results for two faults using a simple diagnostic method because the use of failing scan cells information to locate defect sites is not the focus of this paper. Nevertheless, Table I shows that the two-step method can indeed be used for narrowing down the set of candidate defect sites.
Note that we perform set union here instead of set intersection because the true failing scan cells are not always known exactly, even though they are included in the set of candidate failing scan cells. If only true failing scan cells are included in the candidate set, as in Column 3 of the two-step method, set intersection can be carried out and the number of possible defective sites can be significanlty decreased. For Column 7 of Table I, these numbers are 30 and 64, respectively. Therefore, by identifying a smaller number of failing scan cells, the two-step scheme can also lead to a smaller candidate defect sites.

\section{Application of Two-Step Partitioning to CORE-BASED SOCS}

Fault diagnosis in SOCs has received relatively little attention in the literature, one reason being the lack of availability of 


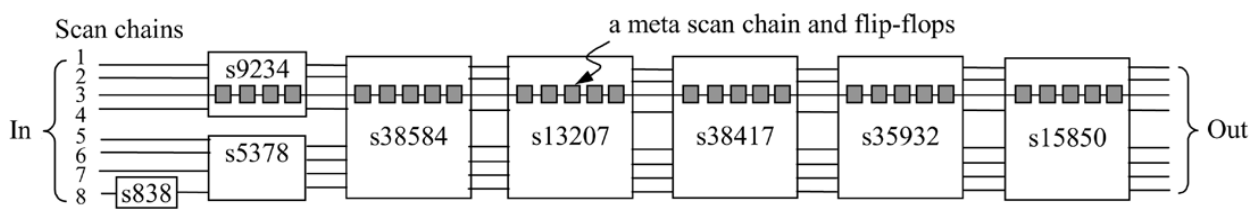

Fig. 10. Scan chain configuration for ISCAS' 89 modules in d695.

internal structural information about intellectual property cores. Here we consider the problem of identifying the error-capturing scan cells in the embedded cores when a test access mechanism (TAM) such as a TestRail [12] is used for test application. The identification of failing scan cells can be applied to all embedded cores with scan architecture.

One possible diagnosis approach is to design a test bus as a TAM and then use a scan cell selection mechanism to identify failing scan cells for one core at a time, as proposed in Sections II and III. However, it suffers from the drawback that since each core may have its own unique scan chain architecture, a single scan chain partitioning configuration for failing scan cell identification cannot accommodate all the cores in the SOC. In addition, this method may require frequent reloading of test patterns to the ATE for the different cores.

An alternative is to use a TestRail and a daisy-chain architecture [12], where "meta" scan chains on the SOC are threaded through the internal scan chains of the embedded cores, i.e., a long scan chain that connects many cores is formed. Test patterns are transported to all the cores and the test responses are transported from the cores using the meta scan chains in a single test session. Test application continues until a core with minimum number of test patterns runs out of all its test patterns. This core is then bypassed and the process repeats for other cores until all the cores run out of test patterns. The failing scan cell identification schemes can be easily applied to this architecture because it requires only a single scan chain partitioning mechanism. Note that the two-step scheme is especially suitable for a daisy-chain architecture. This is because the length of the meta scan chain is changed when a core is dropped from the scan chain, and the configuration of two-step scheme can be easily changed to accommodate the bypassing of cores as discussed in Section IV.

Two-step partitioning is especially suitable for fault diagnosis in SOCs with a daisy-chain architecture. Spot defects on an integrated circuit usually affect a small area of the die, implying that only a small number of cores are likely to be faulty during a test session. If we assume that only one core in the SOC contains failing scan cells, then only a small segment of the meta scan chain contains failing scan cells. As result, the interval-based partitioning approach is especially attractive because the segments containing failing scan cells are clustered within a few small groups when the meta scan chains are partitioned.

In order to highlight the advantages of the proposed diagnosis approach, we present results for two SOCs. The first SOC is crafted by stitching together the six largest ISCAS' 89 benchmarks, and assuming that a single meta scan chain is threaded through the internal scan chains of the cores. The second SOC is a variant of d695 from the ITC'02 SOC Test benchmarks [11]. We only consider the full-scan ISCAS'89 modules in d695. We use a daisy-chain architecture with an eight-bits-wide TAM. The scan chains in the cores are reorganized to construct eight balanced meta scan chains on the SOC, and the cores are daisychained as shown in Fig. 10. For the sake of illustration, we show some scan cells in one meta scan chain using shaded squares. We are unable to use the other ITC' 02 benchmarks because they do not contain sufficient information for carrying out fault simulation.

For each set of experiments, we assume that only one core contains failing scan cells. For each core that is assumed to be faulty, we inject 500 single stuck-at faults, perfrom fault simulation using the test patters from Mintest, and calculate the corresponding DR value. We note that the effect of multiple faults can be viewed similarly with that of single fault, as discussed in Section 3.

A total of eight partitions are used for both SOCs. To determine the number of groups in a partition, our strategy is to use more groups on the longer meta scan chains. The first SOC contains a rather long meta scan chain so we use 32 groups for each partition in this case. For the second SOC, the scan chains are relatively shorter, hence, and the number of groups for each partition is set to eight.

We compare random-selection, deterministic partitioning, and two-step partitioning for the two SOCs, and present simulation results in Figs. 11(a) and (b). The $x$ axis represents the failing core. For the two-step method, only the first partition is interval-based. It can be seen that in most cases, the two step method outperforms the random-selection method and the deterministic approach with monotonic partitioning significantly. In some cases, a 10X improvement is achieved. We omit the results with pruning technique in [7] due to the limited space. We have observed that even with pruning, interval-based partitioning performs significantly better. If we assume that scan chain redesign is not feasible and additional routing overhead cannot be incurred, the results obtained with the two-step method are always better than those obtained with the deterministic approach with nonmonotonic partitioning.

The simulation time of the two-step scheme on the SOC is reasonable. Although the meta scan chain in the SOC is much longer than a scan chain in a single circuit, the CPU time for the simulation is usually less than a few minutes.

Next, as in Fig. 9 of Section V, we inject 200 various types of faults (gate replacement and bridging) into the SOC and repeat the simulation. The results shown in Fig. 12 demonstrate once again that the two-step scheme is compatible with various types of faults.

The two-step method for identification-failing scan cells is not limited to the case of a single failing core. Next, we extend the experiments by assuming that two cores can be failing simultaneously, as shown in Fig. 13. The $x$ axis represents pairs 


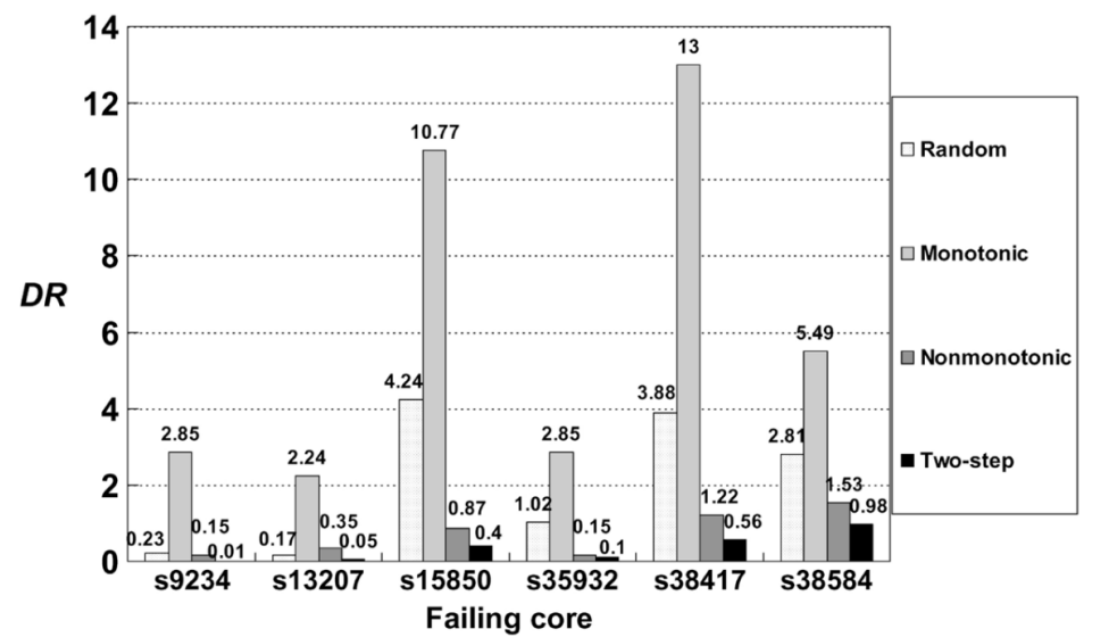

(a)

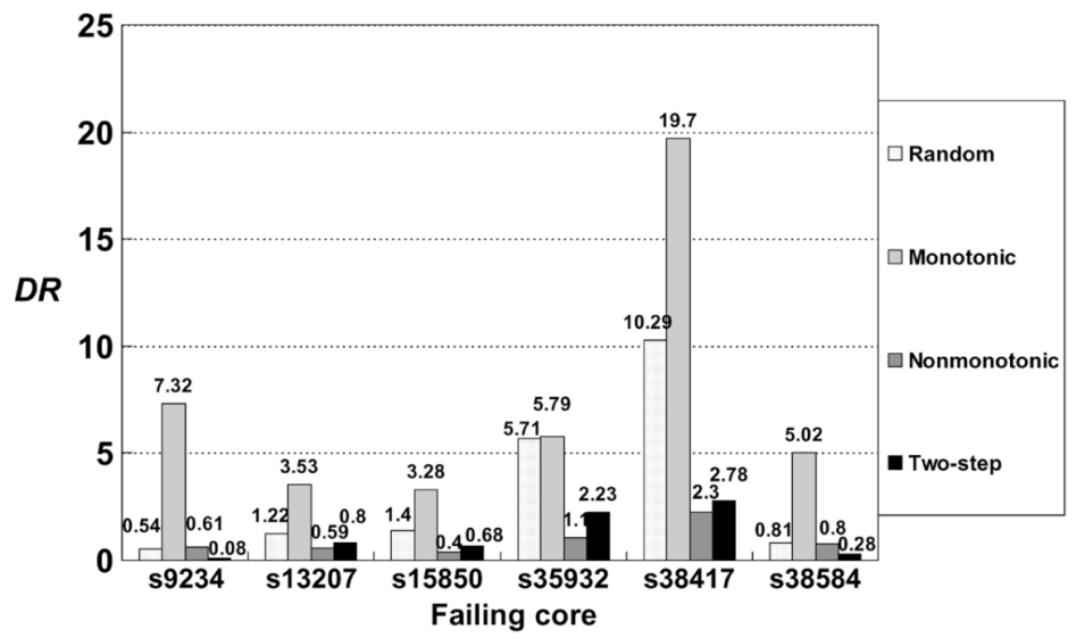

(b)

Fig. 11. SOC diagnostic resolution. (a) Single scan chain. (b) Multiple scan chain.

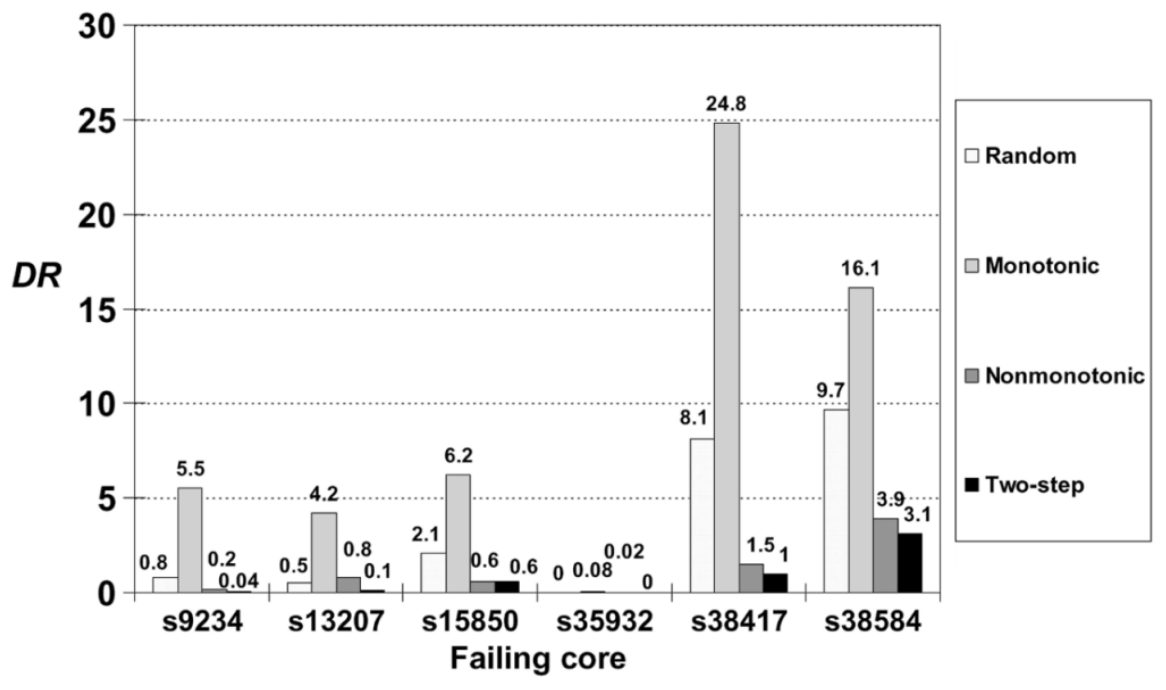

Fig. 12. SOC diagnostic resolution for various types of faults.

of failing cores. Similar results as in Fig. 11 are observed, hence, the proposed scheme appears to be suitable for the case of multiple failing cores.

The diagnosis time is determined to a large extent by the number of partitions required to achieve a desired DR value.
Viewed from another perspective, the increase in diagnostic accuracy implies shorter diagnosis time and a smaller number of partitions required to obtain a desired resolution. In Fig. 14(a), we show the number of partitions needed to obtain a DR value of 0.5 (without pruning) with the three partitioning methods for 


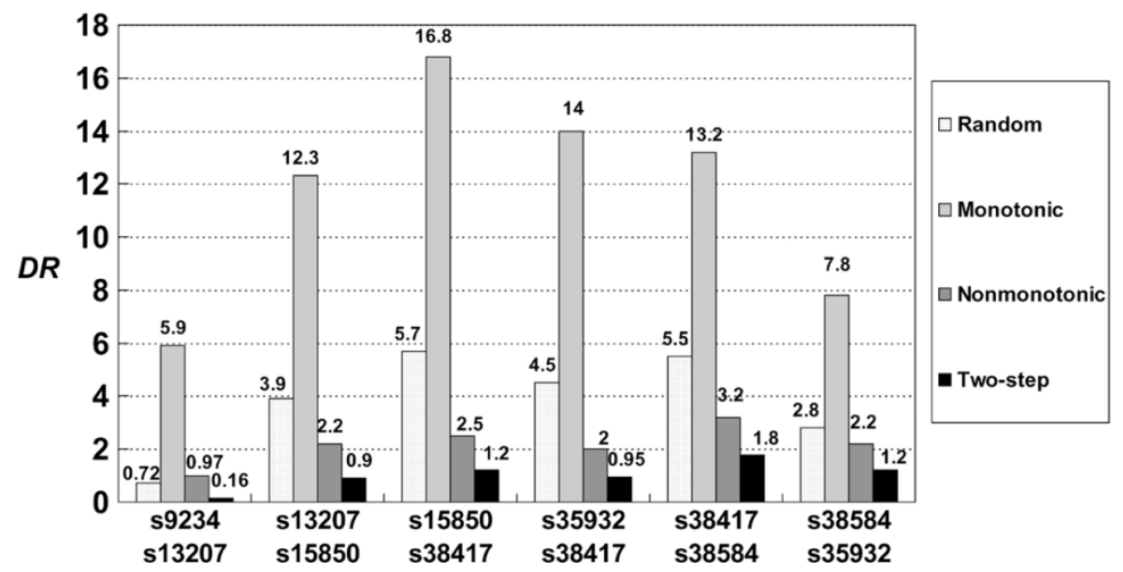

Fig. 13. SOC diagnostic resolution for two failing cores with a single scan chain.

TABLE II

AREa Overheads of DifFERENT SCHEMES IMPLEMENTED Using SYNOPSIS DESIGN ANALYZER

\begin{tabular}{c|c|c|c|c}
\hline & $\begin{array}{c}\text { Random } \\
\text { selection }\end{array}$ & Two-step & $\begin{array}{c}\text { Modulo } \\
\text { arithmetic }\end{array}$ & Galois field \\
\hline $\begin{array}{c}\text { Total area } \\
\text { overhead }\end{array}$ & 716 & 850 & 808 & 378 \\
\hline
\end{tabular}

(a) Area in terms of the number of minimum-sized inverters.

\begin{tabular}{c|c|c|c|c}
\hline & $\begin{array}{c}\text { Random } \\
\text { selection }\end{array}$ & Two-step & $\begin{array}{c}\text { Modulo } \\
\text { arithmetic }\end{array}$ & Galois field \\
\hline $\begin{array}{c}\text { Total area } \\
\text { overhead }\end{array}$ & 1 & 1.19 & 1.13 & 0.53 \\
\hline
\end{tabular}

(b) Nomalized area overhead.

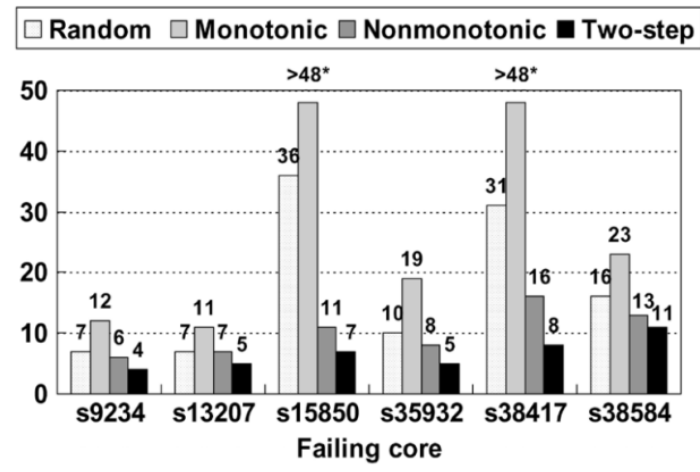

(a)

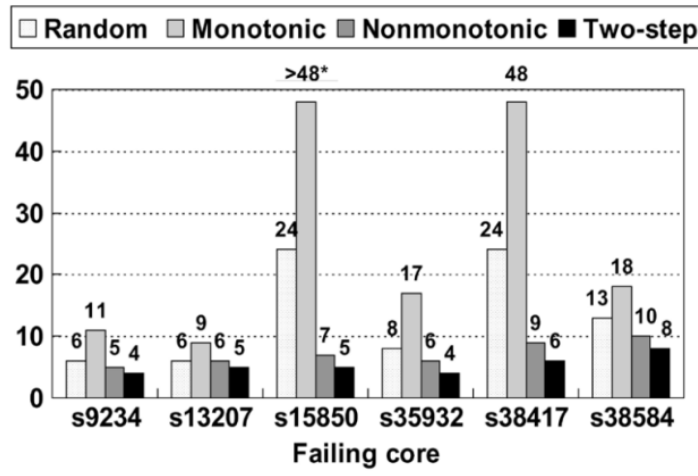

(b)

Fig. 14. Number of partitions required to attain a specific DR value for different failing modules in the SOC with a single scan chain. (a) $\mathrm{DR}=0.5$. (b) $\mathrm{DR}=1$.

the first SOC with a single scan chain. In Fig. 14(b), we show similar results to obtain a DR value of 1 . We observe that the two-step approach requires a smaller number of partitions than the random selection and the deterministic partitioning methods in all cases, with a corresponding reduction in diagnosis time. Recall that the nonmonotonic scheme provides slighly better results than thebtwo-step scheme for s38417 in Fig. 7. However, when the resolution requirement is increased as in Fig. 14 and the circuit is embedded in an SOC, the two-step method outperforms it by as much as $50 \%$.

Finally, we examine the hardware overhead of the different partitioning schemes using Synopsys synthesis tools. We implemented the random-selection scheme, the two-step scheme and the monotonic deterministic schemes using modulo arithmetic and Galois field GF(2) [13] using Synopsis Design Analyzer. The total area, measured by Design Analyzer in terms of the number of minimum-sized inverters, and normalized area overheads (expressed as the percentage of the overhead of the random-selection scheme) are presented in Table II. The area overhead includes all the partitioning logic and the interconnect (calculated using the wire load model in Design Analyzer), as well as the standard BIST shift counter and the BIST pattern counter. The Galois field GF(2) implementation gives the smallest hardware cost. However, as shown in the experimental results, it suffers from low diagnostic resolution. It also requires the number of scan cell groups within a partition be the power of 2 and equal to the number of partitions, which imposes additional constrains. The overheads of the two-step scheme and the modulo arithmetic scheme are comparable and slightly larger than the random-selection scheme. As in GF(2), the scheme based on modulo arithmetic requires that the number of scan cell groups within a partition be a prime number and equal the number of partitions. In view of the improvement in diagnostic 
TABLE III

CONTRIBUtion to THE AREA OVERHEAD OF THE INDIVIDUAL COMPONENTS FOR THE DiFFERENT SCHEMES

\begin{tabular}{c|c|c|c|c}
\hline & $\begin{array}{c}\text { Random } \\
\text { selection }\end{array}$ & Two-step & $\begin{array}{c}\text { Modulo } \\
\text { arithmetic }\end{array}$ & Galois field \\
\hline LFSR/IVR & $66.8 \%$ & $56.3 \%$ & - & - \\
\hline Counters & $31.6 \%$ & $42.6 \%$ & $47.4 \%$ & $93.2 \%$ \\
\hline Others & $1.6 \%$ & $1.1 \%$ & $15.9 \%$ & $6.8 \%$ \\
\hline Adders/comparators & - & - & $36.7 \%$ & - \\
\hline
\end{tabular}

resolution, the additional hardware cost of two-step scheme is reasonable, with the additional benefit that it imposes no restrictions on either scan cell group or partitions.

We did not include the hardware overhead of the nonmonotonic deterministic scheme because it requires scan chain redesign and additional routing overhead, which are difficult to quantify and account for in the calculation of area. As the number of scan cell or partitions increase, the routing overhead becomes a significant limitation for the nonmonotonic deterministic scheme.

In Table III, we show the contribution of the various components to the area overhead for the different schemes. It can be seen that the LFSR and IVR constitute most of the overhead for the first two schemes. The overhead due to the counters is also a major contributor in all cases.

\section{CONCLUSION}

In this paper, we have presented a new two-step partitionbased method for determining failing scan cells in scan-BIST. First, an interval-based partitioning scheme is used to generate a small number of partitions. The remaining partitions are then created using traditional random-selection partitioning. We have shown that two-step partitioning can be implemented with a small amount of hardware. Analytical and experimental results for the ISCAS' 89 benchmarks show that two-step partitioning offers higher diagnostic resolution than competing approaches based on random-selection partitioning and deterministic partitioning. We have shown that this approach is especially suitable for identifying failing scan cells in SOCs with a daisy-chain test access architecture. Experimental results on diagnostic resolution for two SOCs crafted from the ISCAS' 89 benchmarks show that interval-based two-step partitioning significantly outperforms random selection and deterministic partitioning.

\section{REFERENCES}

[1] P. Song, F. Motika, D. R. Knebel, R. F. Rizzolo, and M. P. Kusko, "S/390 G5 CMOS microprocessor diagnostics," in Proc. Int. Test Conf., 1999, pp. 1073-1082.

[2] J. Ghosh-Dastidar, D. Das, and N. A. Touba, "Fault diagnosis in scanbased BIST using both time and space information," in Proc. Int. Test Conf., 1999, pp. 95-102.

[3] T. R. Damarla, C. E. Stroud, and A. Sathaye, "Multiple error detection and identification via signature analysis," J. Electron. Testing: Theory Applicat., vol. 7, no. 3, pp. 193-207, 1995.

[4] C. Liu and K. Chakrabarty, "Failing vector identification based on overlapping intervals of test vectors in a scan-BIST environment," IEEE Trans. Computer-Aided Design, vol. 22, pp. 593-604, May 2003.

[5] J. Rajski and J. Tyszer, "Diagnosis of scan cells in BIST environment," IEEE Trans. Comput., vol. 48, pp. 724-731, July 1999.

[6] J. Ghosh-Dastidar and N. A. Touba, "A rapid and scalable diagnosis scheme for BIST environment with a large number of scan chains," in Proc. VLSI Test Symp., 2000, pp. 79-85.

[7] I. Bayraktaroglu and A. Orailoglu, "Improved fault diagnosis in scanbased BIST via superposition," in Proc. Design Automation Conf., June 2000, pp. 55-58.

[8] - "Deterministic partitioning techniques for fault diagnosis in scanbased BIST," in Proc. Int. Test Conf., Oct. 2000, pp. 273-282.
[9] Y. Wu and S. Adham, "BIST fault diagnosis in scan-based VLSI environment," in Proc. Int. Test Conf., 1996, pp. 48-57.

[10] E. J. Marinissen, R. Arendsen, G. Bos, H. Dingemanse, M. Lousberg, and C. Wouters, "A structured and scalable mechanism for test access to embedded reusable cores," in Proc. Int. Test Conf., 1998, pp. 284-293.

[11] E. J. Marinissen, V. Iyengar, and K. Chakrabarty, "A set of benchmarks for modular testing of SOCs," in Proc. Int. Test Conf., 2002, pp. 519-528.

[12] J. Aerts and E. J. Marinissen, "Scan chain design for test time reduction in core-based ICs," in Proc. Int. Test Conf., 1998, pp. 448-457.

[13] I. Bayraktaroglu and A. Orailoglu, "Cost-effective deterministic partitioning for rapid diagnosis in scan-based BIST," IEEE Design Test Comput., vol. 19, pp. 42-53, Jan./Feb. 2002.

[14] I. Hamzaoglu and J. H. Patel, "Test set compaction algorithms for combinational circuits," in Proc. Int. Conf. Computer-Aided Design, 1998, pp. 283-289.

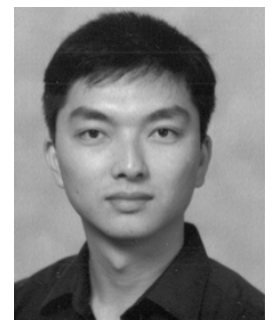

Chunsheng Liu (S'00-M'03) received the B.S and M.S. degrees in electronic engineering from Tsinghua University, Beijing, China, in 1997 and 2000, respectively, and the Ph.D. degree in electrical and computer engineering from Duke University, Durham, NC, in 2003.

$\mathrm{He}$ is currently an Assistant Professor of Computer and Electronics Engineering at University of Nebraska, Lincoln. His research interests include VLSI design, testing, and fault diagnosis. He is currently working in the areas of system-on-chip

design and testing

Prof. Liu is a Member of the Association for Computing Machinery (ACM) and the ACM Special Interest Group on Design Automation.

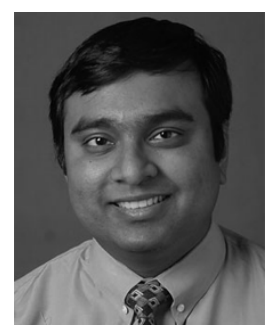

Krishnendu Chakrabarty (S'92-M'96-SM'00) received the B.Tech. degree from the Indian Institute of Technology, Kharagpur, India, in 1990, and the M.S.E. and Ph.D. degrees from the University of Michigan, Ann Arbor, in 1992 and 1995, respectively, all in computer science and engineering.

$\mathrm{He}$ is currently an Associate Professor of Electrical and Computer Engineering at Duke University, Durham, NC. From 2000 to 2002, he was also a Mercator Visiting Professor at the University of Potsdam, Potsdam, Germany. His current research projects include: design and testing of system-on-a-chip integrated circuits; embedded real-time systems; distributed sensor networks; modeling, simulation, and optimization of microelectrofluidic systems; microfluidics-based chip cooling. $\mathrm{He}$ is a coauthor of two books: Microelectrofluidic Systems: Modeling and Simulation (Boca Raton, FL: CRC Press, 2002) and Test Resource Partitioning for System-on-a-Chip (Norwell, MA: Kluwer, 2002), and an editor of SOC (System-on-a-Chip) Testing for Plug and Play Test Automation (Norwell, MA: Kluwer, 2002). He has published over 160 papers in journals and refereed conference proceedings, and holds a US patent in BIST.

Prof. Chakrabarty is a Member of ACM, ACM SIGDA, and Sigma Xi. He is a recipient of the National Science Foundation Early Faculty (CAREER) Award and the Office of Naval Research Young Investigator Award. He received a best paper award at the 2001 Design, Automation, and Test in Europe (DATE) Conference. He is also the recipient of the Humboldt Research Fellowship, awarded by the Alexander von Humboldt Foundation, Germany. He is an Associate Editor of IEEE TRANSACTIONS ON COMPUTER-AIDED DESIGN OF INTEGRATED CIRCUITS AND SYSTEMS, an Editor of the Journal of Electronic Testing: Theory and Applications (JETTA), and a member of the editorial board for Sensor Letters and the Journal of Embedded Computing. He has also served as an Associate Editor of the IEEE TRANSACTIONS ON CIRCUITS AND Systems-PART II: ANAlog AND Digital Signal Processing. He serves as Vice Chair of Technical Activities of the IEEE Test Technology Technical Council and is a Member of the program committees of several IEEE/ACM conferences and workshops. 